\title{
Evidence for Radial Flow of Thermal Dileptons in High-Energy Nuclear Collisions
}

\author{
R. Arnaldi, ${ }^{1}$ K. Banicz, ${ }^{2,3}$ J. Castor, ${ }^{4}$ B. Chaurand,${ }^{5}$ C. Cicalò, ${ }^{6}$ A. Colla, ${ }^{1}$ P. Cortese, ${ }^{1}$ S. Damjanovic,,${ }^{2,3}$ A. David,${ }^{2,7}$
} A. de Falco, ${ }^{6}$ A. Devaux,${ }^{4}$ L. Ducroux,${ }^{8}$ H. En' yo, ${ }^{9}$ J. Fargeix,${ }^{4}$ A. Ferretti, ${ }^{1}$ M. Floris, ${ }^{6}$ A. Förster, ${ }^{2}$ P. Force,${ }^{4}$ N. Guettet, ${ }^{2,4}$ A. Guichard, ${ }^{8}$ H. Gulkanian, ${ }^{10}$ J. M. Heuser, ${ }^{9}$ M. Keil,${ }^{2,7}$ L. Kluberg, ${ }^{2,5}$ C. Lourenço, ${ }^{2}$ J. Lozano, ${ }^{7}$ F. Manso, ${ }^{4}$ P. Martins, ${ }^{2,7}$ A. Masoni, ${ }^{6}$ A. Neves, ${ }^{7}$ H. Ohnishi, ${ }^{9}$ C. Oppedisano, ${ }^{1}$ P. Parracho, ${ }^{2}$ P. Pillot, ${ }^{8}$ T. Poghosyan, ${ }^{10}$ G. Puddu, ${ }^{6}$ E. Radermacher, ${ }^{2}$ P. Ramalhete, ${ }^{2}$ P. Rosinsky, ${ }^{2}$ E. Scomparin, ${ }^{1}$ J. Seixas, ${ }^{7}$ S. Serci, ${ }^{6}$ R. Shahoyan,${ }^{2,7}$ P. Sonderegger, ${ }^{7}$ H. J. Specht, ${ }^{3}$ R. Tieulent, ${ }^{8}$ G. Usai, ${ }^{6}$ R. Veenhof, ${ }^{2}$ and H. K. Wöhri ${ }^{6,7}$

(NA60 Collaboration)

\author{
${ }^{1}$ Università di Torino and INFN, Torino, Italy \\ ${ }^{2}$ CERN, 1211 Geneva 23, Switzerland \\ ${ }^{3}$ Physikalisches Institut der Universität Heidelberg, Heidelberg, Germany \\ ${ }^{4} L P C$, Université Blaise Pascal and CNRS-IN2P3, Clermont-Ferrand, France \\ ${ }^{5}$ LLR, Ecole Polytechnique and CNRS-IN2P3, Palaiseau, France \\ ${ }^{6}$ Università di Cagliari and INFN, Cagliari, Italy \\ ${ }^{7}$ Instituto Superior Técnico, Lisbon, Portugal \\ ${ }^{8} I P N-L y o n$, Université Claude Bernard Lyon-I and CNRS-IN2P3, Lyon, France \\ ${ }^{9}$ RIKEN, Wako, Saitama, Japan \\ ${ }^{10}$ YerPhI, Yerevan Physics Institute, Yerevan, Armenia \\ (Received 26 June 2007; published 16 January 2008)
}

\begin{abstract}
The NA60 experiment at the CERN SPS has studied low-mass dimuon production in $158 \mathrm{~A} \mathrm{GeV} \mathrm{In-In}$ collisions. An excess of pairs above the known meson decays has been reported before. We now present precision results on the associated transverse momentum spectra. The slope parameter $T_{\text {eff }}$ extracted from the spectra rises with dimuon mass up to the $\rho$, followed by a sudden decline above. While the initial rise is consistent with the expectations for radial flow of a hadronic decay source, the decline signals a transition to an emission source with much smaller flow. This may well represent the first direct evidence for thermal radiation of partonic origin in nuclear collisions.
\end{abstract}

DOI: 10.1103/PhysRevLett.100.022302

PACS numbers: 25.75.-q, 12.38.Mh, 13.85.Qk

Among the observables used for the diagnostics of the hot and dense fireball formed in high-energy nuclear collisions, lepton pairs are particularly attractive. In contrast to hadrons, they directly probe the entire space-time evolution of the fireball and freely escape, undisturbed by finalstate interactions. In the invariant mass region $\leq 1 \mathrm{GeV}$, thermal dilepton production is largely mediated by the broad vector meson $\rho(770)$. Because of its strong coupling to the $\pi \pi$ channel and its short lifetime of only $1.3 \mathrm{fm} / \mathrm{c}$, its in-medium properties like mass and width have long been considered as the prime signature for the restoration of chiral symmetry, associated with the QCD phase transition from hadronic to partonic matter [1-3]. In the mass region $>1 \mathrm{GeV}$, thermal dileptons may be produced in either the early partonic or the late hadronic phase of the fireball, based here on hadronic processes other than $\pi \pi$ annihilation.

In contrast to real photons, virtual photons decaying into lepton pairs can be characterized by two variables, mass $M$ and transverse momentum $p_{T}$. Historically, the interest has largely focused on mass, including most recently the first measurement of the space-time averaged $\rho$ spectral function in nuclear collisions [4]. Transverse momentum, on the other hand, contains not only contributions from the spectral function, but encodes the key properties of the expanding fireball, temperature and, in particular, transverse (radial) flow. In the description of hadron $p_{T}$ spectra, the study of collective flow has contributed decisively to the present understanding of the fireball dynamics in nuclear collisions [5,6]. However, while hadrons always receive the full asymptotic flow reached at the moment of decoupling from the flowing medium, lepton pairs are continuously emitted during the evolution, sensing small flow and high temperature at early times, and increasingly larger flow and smaller temperatures at later times. The resulting space-time folding over the temperature-flow history can be turned into a diagnostic tool: the measurement of $p_{T}$ spectra of lepton pairs potentially offers access to their emission region and may thereby differentiate between a hadronic and a partonic nature of the emitting source [7].

Experimentally, dilepton $p_{T}$ spectra associated with direct radiation in the mass region $\leq 1 \mathrm{GeV}$ were previously only investigated by the CERES-NA45 experiment at the CERN SPS [8], but with low statistics. In this Letter, we present the first precise data on such spectra, obtained by 
the NA60 experiment at the CERN SPS for $158 A \mathrm{GeV}$ InIn collisions. Mass spectra in different $p_{T}$ windows [9], uncorrected for acceptance, and preliminary results on acceptance-corrected $p_{T}$ spectra were presented before $[10,11]$.

Details of the NA60 apparatus are contained in $[12,13]$. The different analysis steps follow the same sequence as described in our previous Letter [4], including the critical assessment of the combinatorial background from $\pi$ and $K$ decays by a mixed-event technique [14]. The centralityintegrated net mass spectrum of the final data sample used here is shown in Fig. 1. It contains about 430000 dimuons in the mass range $\leq 1.4 \mathrm{GeV}$. The narrow vector mesons $\omega$ and $\phi$ are completely resolved; the mass resolution at the $\omega$ is $20 \mathrm{MeV}$. Figure 1 also contains the expected contributions from the electromagnetic decays of neutral mesons, i.e., the 2-body decays of the $\eta, \omega$, and $\phi$ resonances and the Dalitz decays of the $\eta, \eta^{\prime}$, and $\omega$. The peripheral data can be described by the sum of these "cocktail" contributions plus the $\rho[4,9]$. This is not possible for the total data as plotted in Fig. 1, due to the existence of a strong excess of pairs. To isolate this excess with a priori unknown characteristics without any fits, the cocktail of the decay sources is subtracted from the data using local criteria which are solely based on the measured mass distribution itself. The $\rho$ is not subtracted. The procedure is illustrated in Fig. 1. Each centrality window is treated

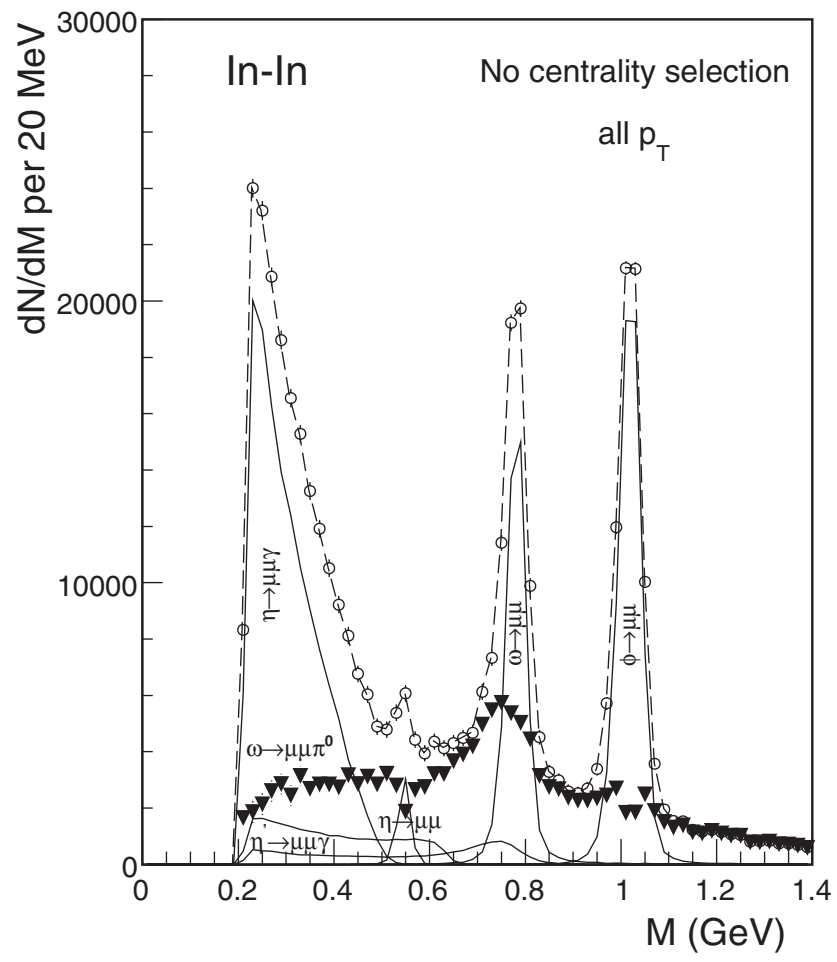

FIG. 1. Isolation of an excess above the electromagnetic decays of neutral mesons (see the text). Total data (open circles), individual cocktail sources (solid line), difference data (thick triangles), sum of cocktail sources and difference data (dashed line). Open charm still contained. separately. The space of $p_{T}$ is subdivided in the range $0 \leq$ $p_{T} \leq 2 \mathrm{GeV}$ into 10 bins of equal width, and each bin is also treated separately. The yields of the narrow vector mesons $\omega$ and $\phi$ are fixed so as to get, after subtraction, a smooth underlying continuum, leading to an accuracy of about $2 \%$ for the $\phi$ and $3 \%-4 \%$ for the $\omega$; at very low $p_{T}$, even an error of $20 \%$ on the $\omega$ would still translate to an error of only $5 \%$ of the excess in the mass region $0.6<$ $M<0.9 \mathrm{GeV}$. The yield of the $\eta$ relative to the $\omega$ and $\phi$, relevant only for masses $\leq 0.4 \mathrm{GeV}$, is fixed from the data at $p T>1.0 \mathrm{GeV}$, profiting from the very high sensitivity of the spectral shape of the Dalitz decay to any underlying admixture from other sources. The yield at lower $p_{T}$, subject to lower statistics due to acceptance, is determined using the NA60 hadron-decay generator GENESIS [15] tuned to the cocktail data. The estimated uncertainty on the $\eta$ subtraction is $\sim 3 \%$, transforming to a systematic error of $\sim 20 \%$ on the excess in the mass region $0.2<M<$ $0.4 \mathrm{GeV}$. Having fixed the three main sources, the $\eta$ twobody and $\omega$ Dalitz decays are then bound as well; the ratio $\eta^{\prime} / \eta$ is assumed to be 0.12 [15]. Open charm, measured to be $0.30 \pm 0.06$ of the total yield in the mass interval $1.2<$ $M<1.4 \mathrm{GeV}$ by NA60 [16], is subtracted throughout (but not yet in Fig. 1), with the spectral shape in $M$ and $p_{T}$ as described by PYTHIA [16]; the resulting uncertainty of the excess is $8 \%$ in this region and $\leq 1 \%$ everywhere else. After subtraction of the meson decays and charm, the remaining sample contains $\sim 150000$ dimuons. The subtracted data for the $\eta, \omega$, and $\phi$ themselves are subject to the same further steps as the excess data and are used later for comparison.

In the last step, the data are corrected for the acceptance of the NA60 apparatus and for the centrality-dependent pair reconstruction efficiencies.

The acceptance shows strong variations with mass and $p_{T}[9,10]$, but is understood on the level of $<10 \%$, mainly based on a detailed study of the peripheral data for the particle ratios $\eta / \omega$ and $\phi / \omega$ [4,9]. In principle, the acceptance correction requires a 3-dimensional grid in $M-p_{T^{-}} y$ space. To avoid large statistical errors in low-acceptance bins, the correction is performed in 2-dimensional $M-p_{T}$ space (with $0.1 \mathrm{GeV}$ bins in mass and $0.2 \mathrm{GeV}$ bins in $p_{T}$ ), using the measured rapidity distribution of the excess as an input [10]. The latter is determined with an acceptance correction in $y$ found, in an iterative way, from Monte Carlo (MC) simulations matched to the data in $M$ and $p_{T}$. Separately for each centrality window, an overlay MC method is used to include the effects of pair reconstruction efficiencies. The resulting values vary from mostly $\geq 0.9$ down to about 0.7 at low mass and low transverse momentum for the highest centrality window. All sources are simulated with a uniform distribution in $\cos \theta_{\mathrm{CS}}$, where $\theta_{\mathrm{CS}}$ is the polar angle of the muons in the Collins-Soper frame, consistent with (yet unpublished) NA60 data for the $\omega$, the $\phi$ and the excess.

Results on acceptance-corrected $p_{T}$ spectra for the mass window $0.6 \leq M \leq 0.9 \mathrm{GeV}$ and the three upper central- 
ities are shown in Fig. 2; equivalent data for other mass windows are contained in [10]. Systematic errors, not contained in Fig. 2, mainly arise from the uncertainties of the combinatorial-background and fake-matches subtraction and range from $10 \%$ to $30 \%$ for semiperipheral up to central collisions at low $p_{T}$, decreasing rapidly to a level of only a few percent at higher $p_{T}$. All other uncertainties are discussed below, in connection with Fig. 4 . The data of the three centrality windows agree within their errors; this also holds for the other mass windows [10].

Figure 3 (upper panel) displays the centrality-integrated data vs transverse mass $m_{T}$, where $m_{T}=\left(p_{T}^{2}+M^{2}\right)^{1 / 2}$, for four mass windows; the $\phi$ is included for comparison. The systematic errors due to the background subtraction are, at low $m_{T}, 30 \%, 25 \%, 15 \%$, and $15 \%$ in the four windows, respectively, falling again rapidly to a level of a few percent at high $m_{T}$; for the $\phi$, they are smaller by a factor of 5. At very low $m_{T}$, a steepening is observed in all four mass windows, reminiscent of pion spectra and opposite to the expectation for radial flow at masses above the pion mass. This increased rise cannot be due to unsubtracted background which increases by a factor of 3 vs centrality, while the data are independent of it (see above). It cannot be due to ill-understood acceptance either, since the $\phi$, placed just between the two upper mass windows, does not show it. While some rise still persists in the peripheral bin, it finally disappears for very peripheral collisions with $4<d N_{\mathrm{ch}} / d \eta<10$ as shown in Fig. 3 (lower panel); the $\omega$ does not show it either. The lines in Fig. 3 are fits with the function $1 / m_{T} d N / d m_{T} \propto$ $\exp \left(-m_{T} / T_{\text {eff }}\right)$, where the effective temperature parameter

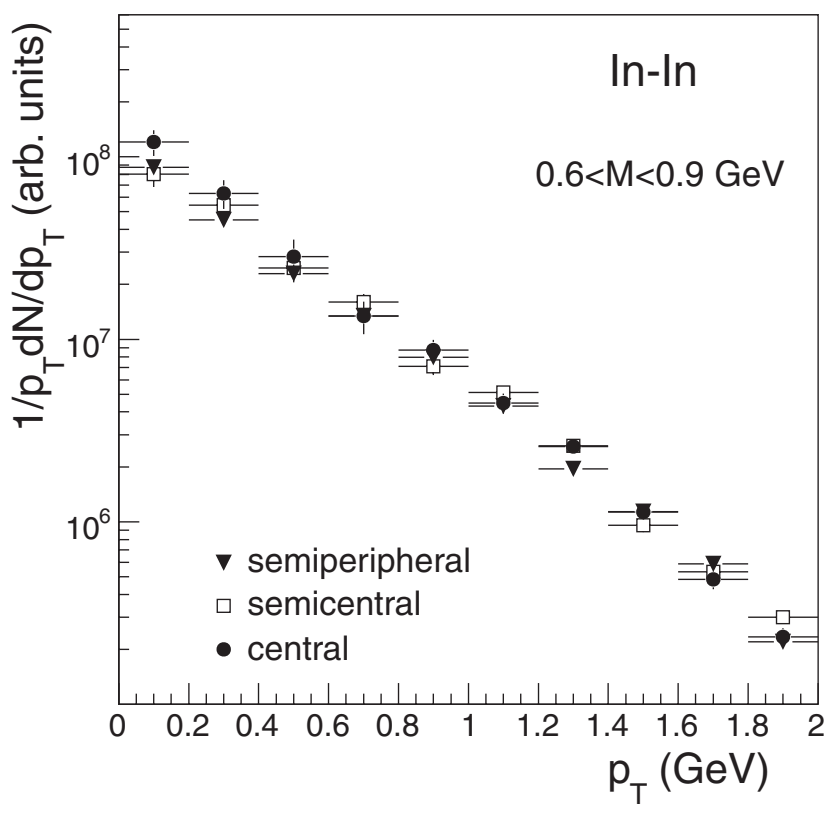

FIG. 2. Transverse momentum spectra of the $\rho$-like mass region for three centrality windows, arbitrarily normalized. The errors are purely statistical; for systematic errors, see the text.
$T_{\text {eff }}$ characterizes the slope of the distributions. For the excess data in Fig. 3 (upper panel), the fits are restricted to the range $0.4<p_{T}<1.8 \mathrm{GeV}$ [roughly $0.1<\left(m_{T}-\right.$ $M)<1.2 \mathrm{GeV}$ ], to exclude the increased rise at low $m_{T}$; for all other spectra, the fits start at zero.

The extracted values of $T_{\text {eff }}$ vs pair mass are summarized in Fig. 4 (open squares), supplemented by a set of further fit values from narrow slices in $M$ (closed triangles). Preliminary NA60 data from an independent analysis [16] of the intermediate mass region ("IMR") $1.16<M<$ $2.56 \mathrm{GeV}$, corrected for the contribution from Drell-Yan pairs, are shown for comparison. Finally, the hadron data obtained as a by-product of the cocktail subtraction procedure are also included; the value for the $\eta$ has been obtained by tuning the GENESIS code [15] to the $\eta$ Dalitz decay and then referring back to the required $T_{\text {eff }}$ of the mother. The errors shown for the low-mass data ("LMR") are purely statistical. Systematic errors enter only to the extent that the slopes of the $p_{T}$ spectra are affected, not

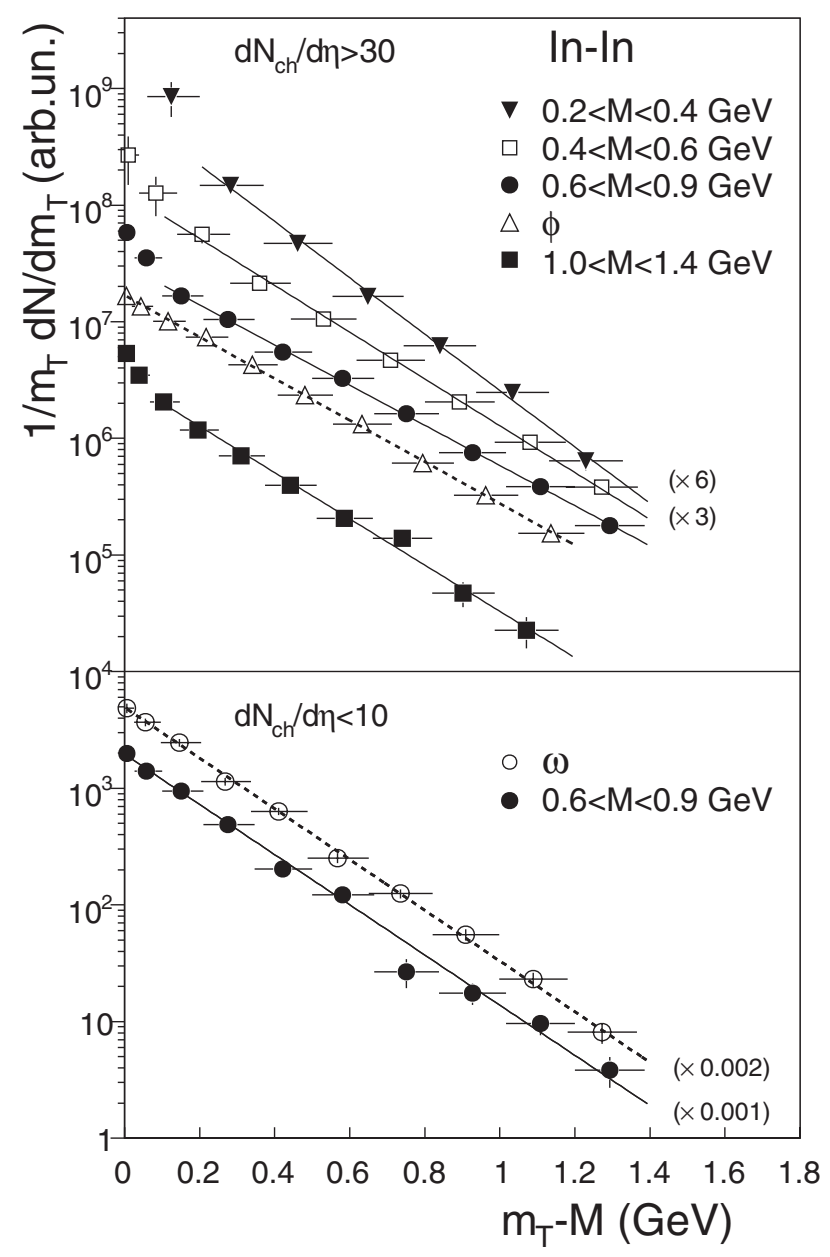

FIG. 3. Upper: Transverse mass spectra of the excess for four mass windows summed over centralities (excluding the peripheral bin), in comparison to the $\phi$. Lower: Transverse mass spectrum of the $\rho$-like mass region (without $\omega$ ) for very peripheral events, in comparison to the $\omega$. The errors are purely statistical; for systematic errors, see the text. 


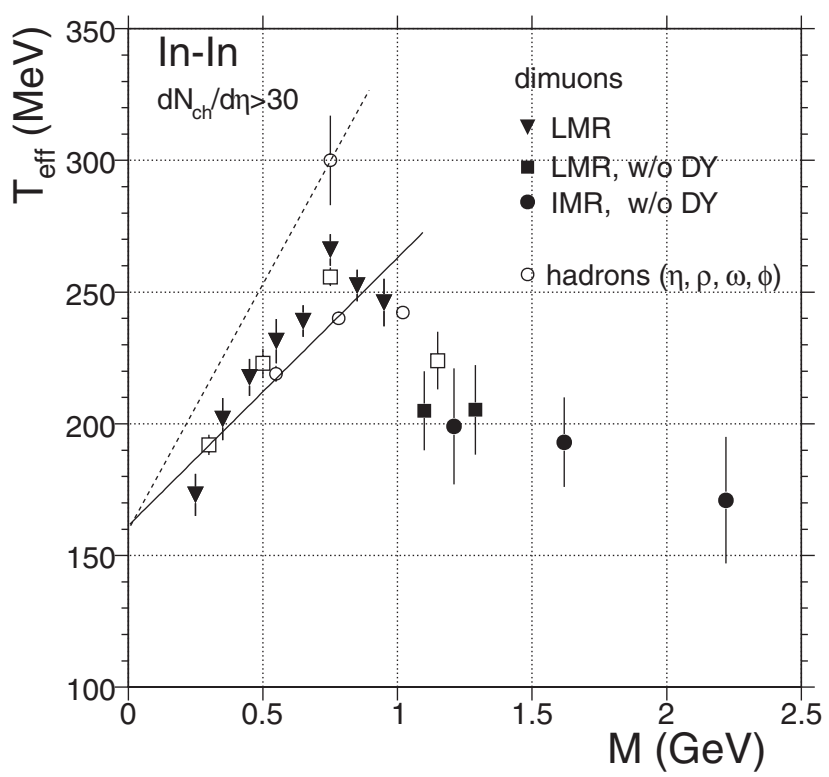

FIG. 4. Inverse slope parameter $T_{\text {eff }}$ vs dimuon mass $M$ for $d N_{\mathrm{ch}} / d \eta>30$. The open squares correspond to the fit lines in Fig. 3. Open charm is subtracted throughout. For explanation of the inserted symbols and the errors, see the text.

their absolute level. The errors due to the background subtraction are about the same as the statistical errors of the fine-bin data $(\sim 7 \mathrm{MeV})$. The errors associated with the subtraction of the decay sources, though significant for the yields (see above), lead to errors of only 1-4 MeV for $T_{\text {eff }}$ (dependent on the source), due to both the mostly local nature of the subtraction and the closeness of $T_{\text {eff }}$ for dimuons and hadrons (see Fig. 4). All other error sources considered - relative acceptance, the sensitivity to the input $y$ distribution, cuts vs no cuts in $y$ (none are used), cuts vs no cuts in $\cos \theta_{\mathrm{CS}}$ (none are used) - also lead to differences considerably smaller than the statistical errors. A correction for Drell-Yan, using an extrapolation down to $M<1 \mathrm{GeV}$ [17], would systematically lower the values by 5-10 MeV, depending on mass.

The results displayed in Fig. 4 can be summarized and interpreted as follows. The slope parameter $T_{\text {eff }}$ rises nearly linearly with mass up to about $270 \mathrm{MeV}$ at the pole position of the $\rho$, followed by a sudden decline to values of 190-200 MeV for masses $>1 \mathrm{GeV}$. The excess yield in the mass region $0.2<M<0.9 \mathrm{GeV}$ has generally been attributed to thermal radiation from the fireball, dominated by pion annihilation $\pi^{+} \pi^{-} \rightarrow \rho \rightarrow \mu^{+} \mu^{-}$, and the NA60 data, before acceptance correction, have directly been interpreted as the space-time averaged inmedium spectral function of the $\rho$ [4]. Following earlier work [3], they are now nearly quantitatively described by the newest theoretical developments [17-19]. The linear rise of $T_{\text {eff }}$ with $M$ over the whole region up to the $\rho$ peak is reminiscent of radial flow of a hadronic source.

While the hadron data show a similar linear rise, their absolute values are surprisingly close to the excess values, contrary to the expectation for the temperature-flow folding expressed in the introduction. The seeming contradiction can be resolved by comparing the (free) $\rho$ itself rather than the other hadrons with the in-medium emission. This $\rho$ is accessible as the peak on the broad continuum (Fig. 1), generally interpreted as the freeze-out $\rho$ without inmedium effects [17-19]. By disentangling the peak from the continuum as done before $[9,10]$, we find $T_{\text {eff }}=300 \pm$ $17 \mathrm{MeV}$ for the peak and $231 \pm 7$ for the underlying continuum in the window $0.6<M<0.9 \mathrm{MeV}$. The high value of the peak, added as a further hadron point into Fig. 4, should then be interpreted as characteristic for the true freeze-out parameters of the fireball, implying the $\eta$, $\omega$, and $\phi$ to freeze-out earlier, due to their smaller coupling to the pions. By modeling a $\rho$ with this temperature, its contribution can be subtracted from the total for each of the finer binned data points in Fig. 4, lowering $T_{\text {eff }}$ by 4-20 MeV depending on the closeness to the pole, and shifting the maximum of the resulting "in-medium" values up to the mass bin just below $1 \mathrm{GeV}$. In any case, the large gap in $T_{\text {eff }}$ between the vacuum $\rho$ and the excess points (corrected or not for the subtraction) restores the expected difference between a freely emitted hadron and its inmedium decay part, making the observed linear rise of $T_{\text {eff }}$ with $M$ now consistent with the expectations for radial flow of a hadronic source (here $\pi \pi \rightarrow \rho$ ) decaying into lepton pairs. Theoretical modeling of our results is under way [17-19], but does not yet describe the data in a satisfactory way.

It is interesting to note that the large gap of $>50 \mathrm{MeV}$ in $T_{\text {eff }}$ between the vacuum $\rho$ and the $\omega$ (same mass) decreases toward the peripheral window, but only closes for the lowest peripheral " $p p$-like" selection $4<d N_{\mathrm{ch}} / d \eta<$ 10 shown in Fig. 3 (lower panel), with $T_{\text {eff }}=198 \pm$ $6 \mathrm{MeV}$ for the $\rho$ and $201 \pm 4 \mathrm{MeV}$ for the $\omega$. This implies that both the "hot $\rho$ " and the low- $m_{T}$ rise discussed before are intimately connected to pions, disappearing together as the $\pi \pi$ contribution to $\rho$ production vanishes (with only the cocktail $\rho$ left).

The sudden decline of $T_{\text {eff }}$ at masses $>1 \mathrm{GeV}$ is the other most remarkable feature of the present data. Extrapolating the lower-mass trend to beyond $1 \mathrm{GeV}$, a jump by about $50 \mathrm{MeV}$ down to a low-flow situation is extremely hard to reconcile with emission sources which continue to be of dominantly hadronic origin in this region. If the rise is due to flow, the sudden loss of flow is most naturally explained as a transition to a qualitatively different source, implying dominantly early, i.e., partonic processes like $q \bar{q} \rightarrow \mu^{+} \mu^{-}$for which flow has not yet built up [18]. While still controversial [17], this may well represent the first direct evidence for thermal radiation of partonic origin, breaking parton-hadron duality for the yield description in the mass domain.

In conclusion, we have found strong evidence for radial flow in the region of thermal dilepton emission which has previously been associated with the $\rho$ spectral function. 
The transition to a low-flow region above may signal a transition from a hadronic to a partonic source.

We are grateful to $H$. van Hees, R. Rapp, T. Renk, and J. Ruppert for useful discussions. We acknowledge support from the BMBF (Heidelberg group) as well as the C. Gulbenkian Foundation, Lisbon, and the Swiss Fund Kidagan (YerPHI group).

[1] R. D. Pisarski, Phys. Lett. 110B, 155 (1982).

[2] G. E. Brown and M. Rho, Phys. Rep. 363, 85 (2002).

[3] R. Rapp and J. Wambach, Adv. Nucl. Phys. 25, 1 (2000).

[4] R. Arnaldi et al., Phys. Rev. Lett. 96, 162302 (2006).

[5] E. Schnedermann, J. Sollfrank, and U.W. Heinz, Phys. Rev. C 48, 2462 (1993).

[6] U.W. Heinz, arXiv:hep-ph/0407360.

[7] K. Kajantie, M. Kataja, L. D. McLerran, and P. V. Ruuskanen, Phys. Rev. D 34, 811 (1986); M. Asakawa, C. M. Ko, and P. Levai, Phys. Rev. Lett. 70, 398 (1993); T. Renk (private communication).
[8] G. Agakichiev et al., Eur. Phys. J. C 41, 475 (2005).

[9] S. Damjanovic et al., Eur. Phys. J. C 49, 235 (2007).

[10] S. Damjanovic et al., Nucl. Phys. A783, 327 (2007).

[11] G. Usai et al., J. Phys. G 34, S233 (2007); J. Seixas et al., J. Phys. G 34, S1023 (2007).

[12] K. Banicz et al., Nucl. Instrum. Methods Phys. Res., Sect. A 546, 51 (2005).

[13] G. Usai et al., Eur. Phys. J. C 43, 415 (2005).

[14] R. Shahoyan et al., Eur. Phys. J. C 43, 209 (2005).

[15] S. Damjanovic, A. De Falco, and H. Wöhri, NA60 Internal Note 2005-1.

[16] R. Shahoyan et al., J. Phys. G 34, S1029 (2007).

[17] H. van Hees and R. Rapp, Phys. Rev. Lett. 97, 102301 (2006); R. Rapp, J. Phys. G 34, S405 (2007); H. van Hees and R. Rapp, J. Phys. G 34, S1051 (2007).

[18] J. Ruppert and T. Renk, arXiv:hep-ph/0612113; J. Phys. G 34, S1047 (2007); J. Ruppert, C. Gale, T. Renk, P. Lichard, and J. I. Kapusta, arXiv:hep-ph/0706.1934.

[19] K. Dusling, D. Teaney, and I. Zahed, Phys. Rev. C 75, 024908 (2007); K. Dusling and I. Zahed, arXiv:hep-ph/ 0701253. 